\title{
Association study of three single-nucleotide polymorphisms in the cyclic adenosine monophosphate response element binding 1 gene and major depressive disorder
}

\author{
YANGE WEI ${ }^{1,2}$, SHUFANG BU ${ }^{2}$, XICAN LIU ${ }^{2}$ and HENGFEN LI ${ }^{1}$ \\ ${ }^{1}$ Department of Psychiatry, First Affiliated Hospital, Zhengzhou University, Zhengzhou, Henan 450052; \\ ${ }^{2}$ Department of Geriatric Neurology, Zhengzhou Central Hospital Affiliated to Zhengzhou University, \\ Zhengzhou, Henan 450007, P.R. China
}

Received May 21, 2014; Accepted December 27, 2014

DOI: $10.3892 /$ etm.2015.2408

\begin{abstract}
Major depressive disorder is a common chronic emotional disorder, and cyclic adenosine monophosphate response element binding protein 1 (CREB1) is hypothesized to play a role in its pathogenesis. The aim of the present study was to investigate the associations between major depressive disorder and relevant single nucleotide polymorphisms (SNPs) in the CREB1 gene. A total of 1,038 subjects of Han Chinese descent were recruited, including 456 patients with major depressive disorder (case group) and 582 healthy volunteers (control group). The frequency distributions of the genotypes and alleles were estimated in the case and control groups, and analyzed for any correlation with major depressive disorder. Three relevant SNP sites in CREB1 were analyzed using quantitative polymerase chain reaction, and statistical analyses were performed to estimate their use as risk factors for major depressive disorder. The analyses revealed that rs2254137 and rs16839883 in CREB1 showed polymorphisms in the sample population, and the genotype and allele frequencies of rs16839883 differed significantly when comparing the patients and healthy controls $(P<0.05)$. No statistically significant differences were detected in the two SNP sites between the male and female patients $(P>0.05)$. Furthermore, no statistically significant differences were detected in rs2254137 genotype and allele distribution when comparing the male and female patients with their corresponding control groups $(P>0.05)$. However, statistically significant differences were observed in the genotype and allele frequencies of rs16839883 when the male and female patients were compared
\end{abstract}

Correspondence to: Dr Hengfen Li, Department of Psychiatry, First Affiliated Hospital, Zhengzhou University, 1 Jianshe East Road, Zhengzhou, Henan 450052, P.R. China

E-mail: lihengfen8106@126.com

Key words: major depressive disorder, gene, cyclic adenosine monophosphate response element binding protein 1, single nucleotide polymorphism with their respective controls $(P<0.05)$. Therefore, the results demonstrated that there is a close correlation between the rs16839883 polymorphism in CREB1 and major depressive disorder, which suggests that this SNP site should be further studied as a potential biomarker for major depressive disorder.

\section{Introduction}

Major depressive disorder is a common, chronic, emotional disease with a high rate of patient suicide and mortality. The condition is clinically characterized by significant and lasting depression, which has a significant impact on the daily life, social interactions and operational capability of patients. Major depressive disorder significantly impacts functionality in life (1), which is a global economic and social problem (2). A crucial step towards finding a treatment for major depressive disorder is to clarify the etiology and pathogenesis of the disease, which remain unclear despite being investigated in previous studies. The etiology of major depressive disorder includes various factors, including genetics, character traits and the environment (3). Genetic factors are considered to be the most important of these (4), since major depressive disorder has a degree of heritability of 0.36-0.66 (5). Previous studies have indicated that major depressive disorder is a complex, polygenetic, hereditary disease $(6,7)$. However, the underlying causes of the disease remain unclear and clinical outcomes are not yet satisfactory.

Cyclic adenosine monophosphate response element binding protein (CREB) may contribute to the pathogenesis of the disease; thus, analyzing the associations of single nucleotide polymorphisms (SNPs) in the CREB gene and major depressive disorder may be significant. CREB is a type of nuclear transcription factor and the association between CREB and major depressive disorder is currently under intensive investigation. Activated CREB can bind to the cAMP response element, a specific regulatory element that is a substrate of phosphorylation for multiple protein kinases, including protein kinase $\mathrm{A}$ (PKA) and C. PKA can regulate the transcription process through autophosphorylation. When cAMP levels in the cytoplasm increase, PKA facilitates the interaction between CREB-binding protein and CREB by 
recognizing the Ser-133 phosphorylation site of CREB, which further enhances transcription. Several neurotransmitters and neural regulators bind to CREB via different kinase pathways, resulting in varying biological effects (8). The activity of cAMP and PKA in the cerebral cortex and hippocampus have been shown to be reduced in depressed rats with acquired helplessness (9), while the expression levels of G protein and CREB are downregulated in the frontal cortex of patients with severe major depressive disorder (10). Moreover, CREB can be overexpressed in the temporal cortex of patients with a long-term history of antidepressant drug use (11). Thus, there is a close association between the pathogenesis and treatment of major depressive disorder and CREB $(9,10)$.

In the present study, case-control comparisons were performed to analyze CREB1 polymorphisms, including rs2254137, rs16839883 and rs2551683, and the association between CREB1 and major depressive disorder.

\section{Subjects and methods}

Major depressive disorder (case group) subjects. Patients were recruited from the Second Hospital Affiliated to Xinxiang Medical University, (Xinxiang, China), Department of Psychiatry, the First Affiliated Hospital of Zhengzhou University, (Zhengzhou, China), the Fifth People's Hospital of Kaifeng (Kaifeng, China) and Luoyang City Fifth People's Hospital (Luoyang, China) between October 2004 and May 2010. All patients were diagnosed with major depressive disorder in accordance with the diagnostic criteria in the 'Diagnostic and Statistical Manual of Mental Disorders in the United States' (DSM-IV; fourth edition) and had no other mental illnesses. The diagnoses were performed by at least two clinicians, including deputy directors, physicians or chief physicians at the hospital. All patients were of the Han Chinese ethnic group, with an age range of 18-60 years, and had a Hamilton Depression Rating Scale (HAM-D, 24-item version) score of $\geq 21$. In addition, the patients had no history of head injury, central nervous system infection or organic mental disorders, and were excluded if their major depressive disorder was the result of the use of psychoactive drugs or other non-addictive substances. All patients participated voluntarily in the study and written informed consent was obtained from each participant or their statutory guardian. Patients were excluded from the study if they exhibited mental retardation, dementia or an alternative mood disorder caused by organic brain or body disease. In addition, patients were excluded if they or any of their relatives were not Han Chinese. The study was conducted with the approval from the Ethics Committee.

Healthy (control group) subjects. Healthy control group subjects were recruited from the Second Hospital Affiliated to Xinxiang Medical University, the School Infirmary in Henan University of Science and Technology (Luoyang, China), the First Affiliated Hospital of Zhengzhou University, Luoyang City Fifth People's Hospital and Kaifeng's Physical Examination Center (Kaifeng, China). The healthy volunteers were all Han Chinese, with an age range of 18-60 years and no history of mental illness. The HAM-D scores for these patients were $<7$. In addition, the patients were all non-smokers, with no history of major bodily disease, alcohol dependence, drug abuse or mental illness in the last three generations of their family. All patients participated in the study voluntarily and written informed consent was obtained from each participant or their statutory guardian. Patients were excluded from the study if they or any of their relatives were not Han Chinese.

Psychiatric assessment. Psychiatric examination of all the subjects in the study was performed simultaneously by two or more clinicians, including deputy directors, physicians or chief physicians, as per the semi-structured procedure of clinical examination in the DSM-IV manual. The HAM-D classification was used to estimate the scale of major depressive disorder. There were five raters, who were psychiatrists with at least four years of clinical experience, and were trained to administer HAM-D assessment and perform scoring. The consistency test was performed simultaneously $(\kappa=0.86-0.94)$.

Subject data. General subject data were collected, including the patient gender, age, nationality, education level, date of birth, occupation, medical history, living environment, smoking habits, alcohol consumption, address, familial situation and contact information. A total of 456 patients with major depressive disorder were recruited, of which 194 were male and 262 were female (age range, $18-60$ years; average, $40.5 \pm 14.5$ years). The course of disease was 24-48 months (average course, $50 \pm 79$ months), and the total HAM-D score range was 21-47 (average score, 28.1 \pm 6.9 ). In addition, a total of 582 healthy volunteers were recruited, of which 327 were male and 255 were female (age range, 18-60 years; average, $33.6 \pm 12.4$ years). No statistically significant differences were identified in gender or age distribution between the case and control groups $(P>0.05)$.

Samples and genotyping. Venous blood $(3 \mathrm{ml})$ was extracted from each subject at the elbow between 08:00am and 10:00am and treated with EDTA to prevent coagulation. The blood samples were centrifuged $(3,000 \mathrm{xg} / \mathrm{min}, 10 \mathrm{~min})$ and the supernatant was extracted and stored at $-70^{\circ} \mathrm{C}$. Total DNA from the supernatant pellet was isolated using the traditional phenolchloroform (Sangon Biotech Co., Ltd, Shanghai, China) method (12).

Based on previous studies (13-15), three SNPs from the CREB1 gene were selected, including rs22541376, rs16839883 and rs2551638. SNP sequences were obtained from the Single Nucleotide Polymorphism Database (http://www.ncbi.nlm.nih. gov/projects/SNP/snp_ref.cgi?rs=387906617), constructed by the National Center for Biotechnology Information and the National Human Genome Research Institute.

SNP genotyping was performed in a 96-well sequential detection system (7300 PRISM; Applied Biosystems Life Technologies, Beijing, China) using a TaqMan Allelic Discrimination assay (Applied Biosystems Life Technologies) (16-18). Each sample was run three times with a simultaneous negative control.

Data processing and analysis. The comparative $\mathrm{Ct}$ method was applied for relative quantification of the genotyping results from the quantitative polymerase chain reaction, using Sequence Detection System Version 2.0 software (Applied Biosystems Life Technologies).

Statistical analysis was performed using SPSS 14.0 software (SPSS, Inc., Chicago, IL, USA). The $\chi^{2}$ test was used to estimate 
Table I. Frequency of genotypes and alleles in the case and control groups.

A, SNP rs 2254137

\begin{tabular}{|c|c|c|c|c|c|}
\hline \multirow[b]{2}{*}{ Groups } & \multicolumn{3}{|c|}{ Genotype, n (\%) } & \multicolumn{2}{|c|}{ Allele, n (\%) } \\
\hline & AA & $\mathrm{AC} / \mathrm{AG}$ & $\mathrm{CC} / \mathrm{GG}$ & A & $\mathrm{C} / \mathrm{G}$ \\
\hline$C(n=438)$ & $171(39)$ & $203(46)$ & $54(15)$ & $545(62)$ & $331(38)$ \\
\hline \multirow[t]{2}{*}{$\mathrm{H}(\mathrm{n}=578)$} & $196(34)$ & $291(50)$ & $91(16)$ & $683(59)$ & $473(41)$ \\
\hline & \multicolumn{3}{|c|}{$\chi^{2}=3.556 \mathrm{P}=0.169$} & \multicolumn{2}{|c|}{$\chi^{2}=2.044 \mathrm{P}=0.153$} \\
\hline
\end{tabular}

B, SNP rs 16839883

\begin{tabular}{|c|c|c|c|c|c|}
\hline \multirow[b]{2}{*}{ Groups } & \multicolumn{3}{|c|}{ Genotype, n (\%) } & \multicolumn{2}{|c|}{ Allele, n (\%) } \\
\hline & $\mathrm{AA}$ & $\mathrm{AC} / \mathrm{AG}$ & $\mathrm{CC} / \mathrm{GG}$ & A & $\mathrm{C} / \mathrm{G}$ \\
\hline$C(n=405)$ & $240(59)$ & $92(23)$ & 73 (18) & $572(71)$ & $238(29)$ \\
\hline \multirow[t]{2}{*}{$\mathrm{H}(\mathrm{n}=562)$} & $324(58)$ & $209(37)$ & $29(5)$ & $857(76)$ & $267(24)$ \\
\hline & \multicolumn{3}{|c|}{$\chi^{2}=139.127 \mathrm{P}<0.001$} & \multicolumn{2}{|c|}{$\chi^{2}=7.729 \mathrm{P}=0.005$} \\
\hline
\end{tabular}

C, patients with major depressive disorder (case group); H, healthy individual (control group); SNP, single nucleotide polymorphism.

differences in the distribution of genotypes and alleles between the case and control groups. Hardy-Weinberg Diagnostics Version 2.0 software (Applied Biosystems Life Technologies) was used to assess whether the genotypes and alleles conformed to the Hardy-Weinberg equilibrium. Odds ratio (OR) and $95 \%$ confidence interval (CI) values were used to estimate the risk of major depressive disorder. $P<0.05$ was considered to indicate a statistically significant difference $(\alpha, 0.05)$.

\section{Results}

Detection of SNPs. DNA samples were collected from the 456 patients with major depressive disorder and 582 healthy volunteers. The case and control subjects exhibited the three SNPs as follows: rs2254137, 438 and 578, respectively; rs16839883, 405 and 562, respectively; and rs2551638, 416 and 293, respectively. All the genotypes of rs2551638 were GG, with no other genotype detected.

Tests for the three SNPs, genotypes and alleles of rs2254137 and rs16839883 conformed to the Hardy-Weinberg equilibrium $(P>0.05)$ in the case and control groups.

Comparison offrequency distributions of the SNPs. Statistically significant differences were observed in the genotype and allele frequency distributions of rs16839883 between the case and control groups $\left(\chi^{2}=139.127, \mathrm{P}<0.001\right.$ and $\chi^{2}=7.729, \mathrm{P}=0.005$, respectively). However, no statistically significant differences were detected between the groups in the allele or genotype frequency distributions of rs2254137 ( $P>0.05$; Table I).

Association analysis of the SNP genotypes with major depressive disorder. Patients with the rs16839883 AG genotype exhibited a lower relative risk of major depressive disorder (OR, 0.496; 95\% CI, 0.372-0.663; $\chi^{2}=22.997 ; P<0.001$ ), whereas patients with the GG genotype showed a higher risk (OR, 4.04; 95\% CI, 2.573-6.348; $\left.\chi^{2}=41.285 ; P<0.001\right)$. No statistically significant difference in major depressive disorder risk was detected between the patients in the case and control groups with the rs2254137 AA, AC and CC genotypes or with the rs16839883 AA genotype $(P>0.05$; Table II).

Comparison of genotype and allele frequencies between male and female patients. A total of 456 patients were recruited in the study, of which 194 were male and 262 were female. No statistically significant differences in the frequencies of genotypes and alleles of rs16839883 and rs2254137 were detected between the male and female patients $(P>0.05$; Table III).

Comparison of genotype and allele frequencies between male/female patients and male/female healthy volunteers. Comparisons of genotype and allele frequencies were performed between male patients and healthy male volunteers, and between female patients and healthy female volunteers. A statistically significant difference was observed between male patients and healthy male volunteers for the rs16839883 genotype $\left(\chi^{2}=19.716, P<0.001\right)$, while no significant difference was detected for rs2254137 ( $P>0.05$; Table IV). Similar results were obtained for female patients and healthy female volunteers (Table V).

\section{Discussion}

Although the rs2551638 polymorphism has been reported in European and African populations (19), the present study revealed that the AA and AG rs2551638 genotypes do not exist in the Han Chinese ethnic group. This conclusion was based on the DNA analysis of 1,038 subjects, including patients with major depressive disorder and healthy subjects (Table I), which 
Table II. Association analysis of the two SNP genotypes in the case and control groups.

A, SNP r2254137

\begin{tabular}{lcccccc}
\hline Genotype & Case group & Control group & OR & 95\% CI & $\chi^{2}$ & $P$-value \\
\hline AA & 171 & 196 & 1.248 & $0.964-1.615$ & 2.843 \\
AC & 203 & 291 & 0.852 & $0.664-1.093$ & 1.595 & 0.092 \\
CC & 64 & 91 & 0.916 & $0.647-1.296$ & 0.247 \\
\hline
\end{tabular}

B, SNP rs 16839883

\begin{tabular}{lcccrrr}
\hline Genotype & Case group & Control group & OR & 95\% CI & $\chi^{2}$ & $P$-value \\
\hline AA & 240 & 324 & 1.068 & $0.824-1.385$ & 0.250 \\
AG & 92 & 209 & 0.496 & $0.372-0.663$ & 22.997 \\
GG & 73 & 29 & 4.041 & $2.573-6.348$ & 41.285 & 0.617 \\
\hline
\end{tabular}

SNP, single nucleotide polymorphism; OR, odds ratio; CI, confidence interval.

Table III. Frequency comparison of genotypes and alleles between male and female patients.

A, SNP rs 2254137

\begin{tabular}{|c|c|c|c|c|c|}
\hline \multirow[b]{2}{*}{ Groups } & \multicolumn{3}{|c|}{ Genotype, n (\%) } & \multicolumn{2}{|c|}{ Allele, n (\%) } \\
\hline & AA & $\mathrm{AC} / \mathrm{AG}$ & $\mathrm{CC} / \mathrm{GG}$ & A & $\mathrm{C} / \mathrm{G}$ \\
\hline$M(n=186)$ & $80(43)$ & $78(42)$ & $28(15)$ & $238(64)$ & $134(36)$ \\
\hline \multirow[t]{2}{*}{$F(n=252)$} & $91(36)$ & $125(50)$ & $36(14)$ & $307(61)$ & $197(39)$ \\
\hline & \multicolumn{3}{|c|}{$\chi^{2}=3.942 \mathrm{P}=0.139$} & \multicolumn{2}{|c|}{$\chi^{2}=0.856 \mathrm{P}=0.355$} \\
\hline
\end{tabular}

B, SNP rs 16839883

\begin{tabular}{|c|c|c|c|c|c|}
\hline \multirow[b]{2}{*}{ Groups } & \multicolumn{3}{|c|}{ Genotype, n (\%) } & \multicolumn{2}{|c|}{ Allele, n (\%) } \\
\hline & AA & $\mathrm{AC} / \mathrm{AG}$ & $\mathrm{CC} / \mathrm{GG}$ & A & $\mathrm{C} / \mathrm{G}$ \\
\hline$M(n=168)$ & $102(61)$ & $40(24)$ & $26(15)$ & $244(73)$ & $92(27)$ \\
\hline \multirow[t]{2}{*}{$F(n=237)$} & $138(58)$ & $52(22)$ & $47(20)$ & $328(69)$ & $146(31)$ \\
\hline & \multicolumn{3}{|c|}{$\chi^{2}=3.244 \mathrm{P}=0.198$} & \multicolumn{2}{|c|}{$\chi^{2}=1.109 \mathrm{P}=0.292$} \\
\hline
\end{tabular}

M, male patients; F, female patients; SNP, single nucleotide polymorphism.

indicates that the Han Chinese population may not have the rs2551638 polymorphism. All the examined population were of the GG genotype, which suggests that rs2551638 may vary between ethnic groups and that this site may not be involved in the pathogenesis of major depressive disorder. No significant evidence was found to suggest an association between rs2551638 and major depressive disorder (Table II).

Polymorphic analysis of CREB1 rs16839883 and rs2254137 in 456 patients and 582 healthy individuals revealed that the frequency distributions of the genotypes and alleles conformed to the Hardy-Weinberg equilibrium, suggesting that the subject population was reliable and scientifically robust. The frequency distributions of CREB1 rs16839883, rs2551638 and rs2254137 were estimated in the Han Chinese population using the $\chi^{2}$ test, and it was found that rs 2551638 was of the GG genotype with no polymorphism.

No statistically significant differences were detected in the rs2254137 genotype and allele distributions between the case and control groups $(P>0.05)$, while a statistically significant difference was detected between the groups for the rs16839883 genotype distribution $\left(\chi^{2}=139.127, P<0.001\right)$. In addition, a statistically significant difference was observed between the two groups in the A/G allele distribution of the rs16839883 CREB1 SNP $\left(\chi^{2}=7.729, P=0.005\right)$. These observations 
Table IV. Frequency comparison of genotypes and alleles between male patients and healthy individuals.

A, SNP rs 2254137

\begin{tabular}{|c|c|c|c|c|c|}
\hline \multirow[b]{2}{*}{ Groups } & \multicolumn{3}{|c|}{ Genotype, n (\%) } & \multicolumn{2}{|c|}{ Allele, n (\%) } \\
\hline & AA & $\mathrm{AC} / \mathrm{AG}$ & $\mathrm{CC} / \mathrm{GG}$ & A & $\mathrm{C} / \mathrm{G}$ \\
\hline$M(n=186)$ & $80(43)$ & $78(42)$ & $28(15)$ & $238(64)$ & $134(36)$ \\
\hline \multirow[t]{2}{*}{$\mathrm{H}(\mathrm{n}=325)$} & $107(32.9)$ & $162(49.8)$ & $56(17.3)$ & $376(57.8)$ & $274(42.2)$ \\
\hline & \multicolumn{3}{|c|}{$\chi^{2}=5.270 P=0.074$} & \multicolumn{2}{|c|}{$\chi^{2}=3.710 P=0.055$} \\
\hline
\end{tabular}

B, SNP rs 16839883

\begin{tabular}{|c|c|c|c|c|c|}
\hline \multirow[b]{2}{*}{ Groups } & \multicolumn{3}{|c|}{ Genotype, n (\%) } & \multicolumn{2}{|c|}{ Allele, n (\%) } \\
\hline & AA & $\mathrm{AC} / \mathrm{AG}$ & $\mathrm{CC} / \mathrm{GG}$ & A & $\mathrm{C} / \mathrm{G}$ \\
\hline$M(n=168)$ & $102(61)$ & $40(24)$ & $26(15)$ & $244(73)$ & $92(27)$ \\
\hline \multirow[t]{2}{*}{$\mathrm{H}(\mathrm{n}=319)$} & $177(55.5)$ & $124(38.9)$ & $18(5.6)$ & $478(74.9)$ & $160(251)$ \\
\hline & \multicolumn{3}{|c|}{$\chi^{2}=19.716 P<0.001$} & \multicolumn{2}{|c|}{$\chi^{2}=0.608 P=0.435$} \\
\hline
\end{tabular}

M, male patients with major depressive disorder; H, healthy male subjects; SNP, single nucleotide polymorphism.

Table V. Frequency comparison of genotypes and alleles between female patients and healthy individuals.

A, SNP rs 2254137

\begin{tabular}{|c|c|c|c|c|c|}
\hline \multirow[b]{2}{*}{ Groups } & \multicolumn{3}{|c|}{ Genotype, n (\%) } & \multicolumn{2}{|c|}{ Allele, n (\%) } \\
\hline & AA & $\mathrm{AC} / \mathrm{AG}$ & $\mathrm{CC} / \mathrm{GG}$ & A & $\mathrm{C} / \mathrm{G}$ \\
\hline$F(n=252)$ & $91(36)$ & $125(50)$ & $36(14)$ & 307 (61) & $197(39)$ \\
\hline \multirow[t]{2}{*}{$H(n=253)$} & $89(35.2)$ & $129(51)$ & $35(13.8)$ & $307(60.7)$ & 199 (39.3) \\
\hline & \multicolumn{3}{|c|}{$\chi^{2}=0.097 P=0.953$} & \multicolumn{2}{|c|}{$\chi^{2}=0.006 P=0.938$} \\
\hline
\end{tabular}

B, SNP rs 16839883

\begin{tabular}{|c|c|c|c|c|c|}
\hline \multirow[b]{2}{*}{ Groups } & \multicolumn{3}{|c|}{ Genotype, n (\%) } & \multicolumn{2}{|c|}{ Allele, n (\%) } \\
\hline & AA & $\mathrm{AC} / \mathrm{AG}$ & $\mathrm{CC} / \mathrm{GG}$ & $\mathrm{A}$ & $\mathrm{C} / \mathrm{G}$ \\
\hline$F(n=237)$ & $138(58)$ & $52(22)$ & $47(20)$ & $328(69)$ & $146(31)$ \\
\hline \multirow[t]{2}{*}{$\mathrm{H}(\mathrm{n}=243)$} & $147(60.5)$ & $85(35)$ & $11(4.5)$ & $379(78)$ & $107(22)$ \\
\hline & \multicolumn{3}{|c|}{$\chi^{2}=30.508 P<0.001$} & \multicolumn{2}{|c|}{$\chi^{2}=9.542 P=0.002$} \\
\hline
\end{tabular}

F, female patients with major depressive disorder; $\mathrm{H}$, healthy female subjects; SNP, single nucleotide polymorphism.

indicate that the CREB1 gene may be a relevant susceptibility gene for major depressive disorder. Downregulation of CREB1 expression may be influenced by a variation in the $A / G$ allele, which indicates that reduced expression of CREB1 may contribute to the pathogenesis of major depressive disorder.

Liu et al revealed an association in haplotype between two CREB1 SNPs (rs10932201 and rs6740584) and depression (20), which suggested that CREB1 may play a role in the pathogenesis of the disorder (21-25). Serretti et al found that CREB1 polymorphisms are associated with drug tolerance through the detection of five SNPs in CREB1 (rs2709376, rs2253206, rs7569963, rs7594560 and rs4675690) (26). In addition, previous studies identified reduced expression levels of CREB and phosphorylated-CREB (p-CREB) in the encephalic region of a cadaver specimen, including the temporal lobe, frontal 
orbital plane and the hippocampus (27-29). The expression of CREB has been observed to significantly decrease compared with the control subjects $(30,31)$ and CREB is upregulated by chronic antidepressant treatment, with increased CREB levels in rodent models resulting in antidepressant-like behaviors (32-34). Thus, it is reasonable to suggest that the CREB/p-CREB may be involved in the etiology of depression.

In conclusion, the results of the present study were consistent with those of previous studies, and further support the association between CREB and the pathogenesis of major depressive disorder. Therefore, the CREB1 gene may be a potential target gene for antidepressive therapy.

\section{Acknowledgements}

This study was supported by a grant from the National Natural Science Foundation of China (no. 81371494).

\section{References}

1. Lopez AD, Mathers CD, Ezzati M, Jamison DT and Murray CJ: Global and regional burden of disease and risk factors, 2001: systematic analysis of population health data. Lancet 367: 1747-1757, 2006.

2. Spiessl H, Hübner-Liebermann B and Hajak G: Depression, a widespread disease. Epidemiology, care situation, diagnosis, therapy and prevention. Dtsch Med Wochenschr 131: 35-40, 2006 (In German).

3. Mamdani F, Alda M, Grof P, Young LT, Rouleau G and Turecki G: Lithium response and genetic variation in the CREB family of genes. Am J Med Genet B Neuropsychiatr Genet 147B: 500-504, 2008.

4. Risch N, Herrell R, Lehner T, et al: Interaction between the serotonin transporter gene (5-HTTLPR), stressful life events, and risk of depression: a meta-analysis. JAMA 301: 2462-2471, 2009.

5. Kendler KS, Gatz M, Gardner CO and Pedersen NL: Personality and major depression: a Swedish longitudinal, population-based twin study. Arch Gen Psychiatry 63: 1113-1120, 2006.

6. Terracciano A, Tanaka T, Sutin AR, et al: Genome-wide association scan of trait depression. Biol Psychiatry 68: 811-817, 2010.

7. Cryan JF and Mombereau C: In search of a depressed mouse: utility of models for studying depression-related behavior in genetically modified mice. Mol Psychiatry 9: 326-357, 2004

8. Gaspar L, van de Werken M, Johansson AS, Moriggi E, Owe-Larsson B, Kocks JW, Lundkvist GB, Gordijn MC and Brown SA. Human cellular differences in cAMP--CREB signaling correlate with light-dependent melatonin suppression and bipolar disorder. Eur J Neurosci 40: 2206-2215, 2014.

9. Meyer J, Wiedemann P, Okladnova O, et al: Cloning and functional characterization of the human norepinephrine transporter gene promoter. J Neural Transm 105: 1341-1350, 1998.

10. Zhang HT, Whisler LR, Huang Y, Xiang Y and O'Donnell JM: Postsynaptic alpha-2 adrenergic receptors are critical for the antidepressant-like effects of desipramine on behavior Neuropsychopharmacology 34: 1067-1077, 2009.

11. $\mathrm{Hu}$ YF, Caron MG and Sieber-Blum M: Norepinephrine transport-mediated gene expression in noradrenergic neurogenesis. BMC Genomics 10: 151, 2009.

12. Brummett BH, Babyak MA, Williams RB, Harris KM, Jiang R, Kraus WE, Singh A,Costa PT, Georgiades A and Siegler IC: A Putatively Functional Polymorphism in the HTR2C Gene is Associated with Depressive Symptoms in White Females Reporting Significant Life Stress. PLoS One 9: e114451, 2014.

13. Fabbri C, Souery D, Calati R, et al: Genetics of psychotropic medication induced side effects in two independent samples of bipolar patients. J Neural Transm 122: 43-58, 2015.

14. Li M, Luo XJ, Rietschel M, et al: Allelic differences between Europeans and Chinese for CREB1 SNPs and their implications in gene expression regulation, hippocampal structure and function, and bipolar disorder susceptibility. Mol Psychiatry. 19: 452-461, 2014.
15. Calati R, Crisafulli C, Balestri M, et al: Evaluation of the role of MAPK1 and CREB1polymorphisms on treatment resistance, response and remission in mood disorder patients. Prog Neuropsychopharmacol Biol Psychiatry. 1;44:271-278, 2013.

16. Bae JS, Kim JY, Park BL, et al: Investigating the potential genetic association between RANBP9 polymorphisms and the risk ofschizophrenia. Mol Med Rep Dec 4, 2014 (Epub ahead of print). doi: $10.3892 / \mathrm{mmr} .2014 .3045$.

17. Zhong X, Zhang L, Han S, An Z and Yi Q: Case control study of association between the ANK3 rs10761482 polymorphism and schizophrenia in persons of Uyghur nationality living in Xinjiang China. Shanghai Arch Psychiatry 26: 288-293, 2003.

18. Jitoku D, Yamamoto N, Iwayama Y, et al: Association study of H2AFZ with schizophrenia in a Japanese case-control sample. J Neural Transm. Nov 13, 2014 (Epub ahead of print).

19. Oelke M, Hedlund P, Albrecht K, et al: Expression of cAMP and cGMP-phosphodiesterase isoenzymes 3, 4, and 5 in the human clitoris: immunohistochemical and molecular biology study. Urology 67: 1111-1116, 2006.

20. Liu XH: Preliminary study on the genetic effects and genetic mode of first-episode depression, as well as association and expression study of CREB1, BDNF gene in major depression. Unpublished PhD thesis. Fudan University, Shanghai, 2006 (In Chinese).

21. Liu XH, Xu YF, Cui DH, et al: Association of cyclic adenosine monophosphate response element-binding protein gene and major depressive disorder. Zhonghua Yi Xue Yi Chuan Xue Za Zhi 27: 263-266, 2010 (In Chinese).

22. Turner CA, Thompson RC, Bunney WE, et al: Altered choroid plexus gene expression in major depressive disorder. Front Hum Neurosci 8: 238, 2014

23. Li M, Luo XJ, Rietschel M, et al: MooDS Bipolar Consortium, Swedish Bipolar Study Group, Alzheimer's Disease Neuroimaging Initiative, ENIGMA Consortium and CHARGE Consortium: Allelic differences between Europeans and Chinese for CREB1 SNPs and their implications in gene expression regulation, hippocampal structure and function, and bipolar disorder susceptibility. Mol Psychiatry 19: 452-461, 2014.

24. Lipina TV, Fletcher PJ, Lee FH, Wong AH and Roder JC: Disrupted-in-schizophrenia-1 Gln31Leu polymorphism results in social anhedonia associated with monoaminergic imbalance and reduction of CREB and $\beta$-arrestin-1,2 in the nucleus accumbens in a mouse model of depression. Neuropsychopharmacology 38 : 423-436, 2013.

25. Wang H, Zhang Y and Qiao M: Mechanisms of extracellular signal-regulated kinase/cAMP response element-binding protein/brain-derived neurotrophic factor signal transduction pathway in depressive disorder. Neural Regen Res 8: 843-852, 2013.

26. Serretti A, Chiesa A, Calati R, et al: A preliminary investigation of the influence of CREB1 gene on treatment resistance in major depression. J Affect Disord 128: 56-63, 2011.

27. Burcescu I, Wigg K, King N, et al: Association study of CREB1 and childhood-onset mood disorders. Am J Med Genet B Neuropsychiatr Genet 137B: 45-50, 2005.

28. Calati R, Crisafulli C, Balestri M, et al: Evaluation of the role of MAPK1 and CREB1 polymorphisms on treatment resistance, response and remission in mood disorder patients. Prog Neuropsychopharmacol Biol Psychiatry 44: 271-278, 2013.

29. Martini C, Da Pozzo E, Carmassi C, et al: Cyclic adenosine monophosphate responsive element binding protein in post-traumatic stress disorder. World J Biol Psychiatry 14: 396-402, 2013.

30. Dowlatshahi D, MacQueen GM, Wang JF, Reiach JS and Young LTG: Protein-coupled cyclic AMP signaling in postmortem brain of subjects with mood disorders: effects of diagnosis, suicide, and treatment at the time of death. J Neurochem 73: 1121-1126, 1999.

31. Yamada S, Yamamoto M, Ozawa H, Riederer P and Saito T: Reduced phosphorylation of cyclic AMP-responsive element binding protein in the postmortem orbitofrontal cortex of patients with major depressive disorder. J Neural Transm 110: 671-680,2003

32. Koch JM, Hinze-Selch D, Stingele K, et al: Changes in CREB phosphorylation and BDNF plasma levels during psychotherapy of depression. Psychother Psychosom 78: 187-192, 2009.

33. Blendy JA. The role of CREB in depression and antidepressant treatment. Biol Psychiatry 59: 1144-1150, 2006.

34. Qi X, Lin W, Li J, Li H, Wang W, Wang D and Sun M: Fluoxetine increases the activity of the ERK-CREB signal system and alleviates the depressive-like behavior in rats exposed to chronic forced swim stress. Neurobiol Dis 31: 278-285, 2008. 